\title{
Improving the Tolerability and Efficiency of Electrocardiograms in Psychiatric Hospitals: A Pilot Study
}

\author{
Dr Thomas Wand* \\ St Ann's Acute Psychiatric Hospital, UK
}

*Corresponding author: Thomas Wand, St Ann's Acute Psychiatric Hospital, Dorset, UK.

Received Date: October 16, 2020

Published Date: November 12, 2020

\section{ECGs in Psychiatry}

Many of the anti-psychotic drugs have the side-effect of corrected QT interval (QTc) prolongation. QTc prolongation is associated with ventricular arrhythmias, Torsade de pointes and sudden death. Therefore, before starting anti-psychotics, it is important to perform an ECG to measure the QTc. Furthermore, drugs such as clozapine have common side-effects such as tachycardia. Due to this, NICE guidelines have stated it is good practice to have an ECG "performed at the earliest opportunity, within a 24 hour period".

\section{Challenges}

12-lead ECGs are a non-invasive but intimate investigation requiring the exposure of the torso and in some cases the shaving of chest hair. Patients often find this embarrassing or uncomfortable. It is often difficult to convey the necessity of an ECG to a psychiatric patient who is seeking help with psychiatric symptoms, not cardiac symptoms. These factors lead to frequent patient refusal. The most challenging psychiatric cohort to obtain an ECG from is the acutely manic or psychotic patient. There is firm rationale for improving the acceptability, tolerability, and convenience of any necessary investigation, including ECGs.

\section{New Developments and Technologies}

AliveCor has developed a single lead ECG device, called Kardia Mobile (henceforth referred to as Kardia), easily purchasable on the internet for approximately $£ 100$. Kardia is a small, thin rectangle, with two mental electrodes. It measures a single ECG lead by placing two fingers on either pad, for 30 seconds. The device connects via Bluetooth to a smart device through a downloadable app. A PDF is generated which can be saved or emailed to the appropriate person. Significantly less training is required for Kardia and could be more widely used with less burden on organizational training.

Aims

1. Establish a need for improvements in the 12-lead ECG

2. Compare the patient rating of 12-lead ECG vs Kardia

3. Compare the time duration to perform a 12-lead ECG vs Kardia

4. Compare the accuracy of Kardia vs 12-lead ECG, examining

a) Heart Rate (HR)

b) Correct QT Interval (QTc)

c) PR interval

d) Wave morphology

\section{Inclusion criteria}

1) Any adult admitted to the acute psychiatric admissions unit.

\section{Exclusion criteria}

1) Any patient with cardiac symptomology, such as chest pain. 
2) Patients with a significant cardiac past medical history

3) Patient who are highly agitated.

\section{Methodology}

The pilot study was performed on the Seaview Ward (acute psychiatric admission unit) at St Ann's Hospital, Poole, Dorset, during November 2019.

Cycle 1: The Gold Standard - Demonstrating Area for Improvement

1. 10 new admissions to acute psychiatric ward were invited to have an ECG.

\section{Measured outcomes}

Duration of 12-lead ECG- defined as the time from beginning to remove the item of clothing to when the clothing had been reapplied.

\section{Subjective experience}

Patients were asked to rate their experience of a 12-lead ECG on a 10-point likert scale. Verbal feedback was also documented.

Cycle 2: Gold Standard vs Kardia- Demonstrating Equivalence

1. 10 new admissions to acute psychiatric ward were invited to have both a 12-lead ECG and a Kardia ECG, allowing direct comparisons of objective results such as heart rate, QTc, PR interval, duration of ECG, and subjected measurements such as general morphology and patient experience.

Cycle 3: Kardia alone- Demonstrating Independence

1. 10 new admissions were invited to have a Kardia ECG only. Repeat of Cycle 1, with substituting Gold Standard with Kardia.

\section{Result}

Kardia dramatically outperformed the 12- lead ECG in time efficiency, with high statistical significance (Figure 1). In the 12lead, the majority of the time was spent in removing patients' clothes and applying sticky pads. Figures 2, 3 \& 4 compare the objective data points generated by both Kardia and the 12-lead ECG on the same individua. The value generated by Kardia closely match those of the 12-lead ECG. The standard deviation bars overlap, and p-values are greater than 0.05 , therefore, we can assume there is no statistically significant difference between 12lead ECGs and Kardia in measuring QTc, PR interval or heart rate. Patients demonstrated a slight preference for Kardia in terms of their general experience (Figure 5). However, this was not statically significant. Of note, one patient refused to have a 12-lead ECG but accepted a Kardia ECG. Verbal feedback from patients: "this is much better than the other machine", "Kardia is more easy and less time consuming". Figure 6 shows a typical Kardia ECG, QRS morphology and T-waves, with some background interference. A value for heart rate was automatically generated. PR interval and QTc were manually calculated. For statistical analysis, a paired t test was used throughout. Raw data available on request.

\section{Discussion}

The pilot study demonstrates that the Kardia ECG is significantly faster than the 12-lead ECG, saving 281 seconds, on average, per person. St Ann's Seaview ward sees $\sim 700$ new admissions every year. This would save around 54 hours every year. With the current constraints and pressures on doctors' and nurses' time, the saving of more than 2 days of wasted time is significant, giving clinical staff greater time to focus on other aspects of patient care. Crucially, it appears that the key data points generated are not significantly different from the gold standard, which validates Kardia in measuring QTc, HR, and PR intervals. As far as the author is aware, this is the first time Kardia has been used and validated in this setting. Additionally, general morphology of the QRS complex was similar to that of lead I on a 12- lead ECG. Both Kardia and the gold standard had similar completion rates of $90 \%$. The authors would continue to recommend the exclusion criteria used in this study, as Kardia does not give sufficient information to diagnose or exclude other pathology.

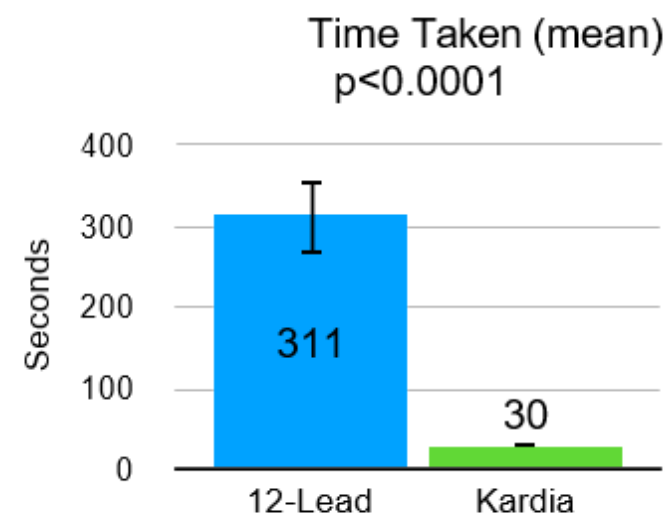

Figure 1: Average Time to Complete ECG. 


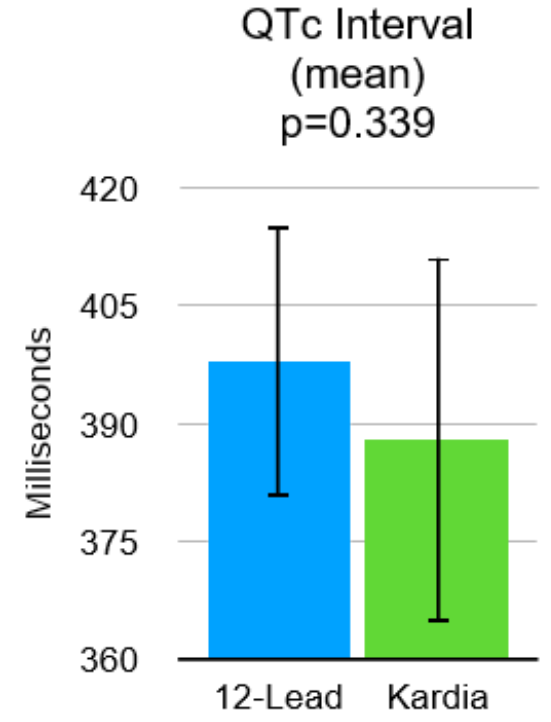

Figure 2: Comparing values of 12-lead ECG.

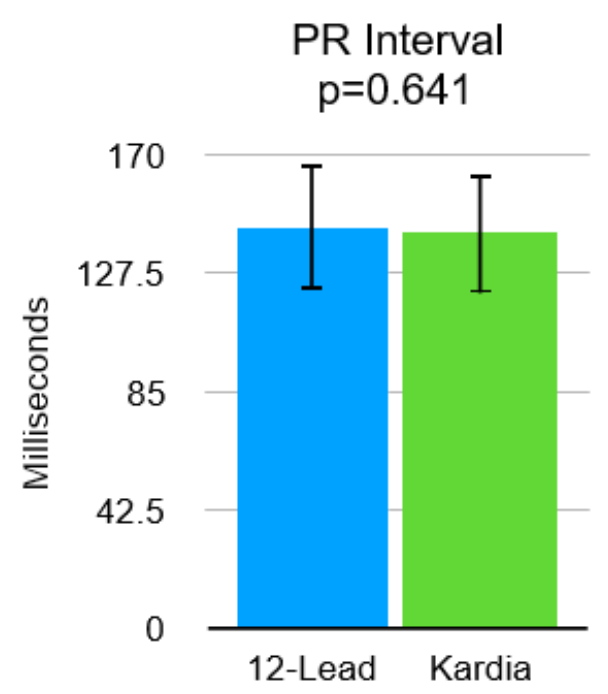

Figure 3: Kardia in Measuring QTC.

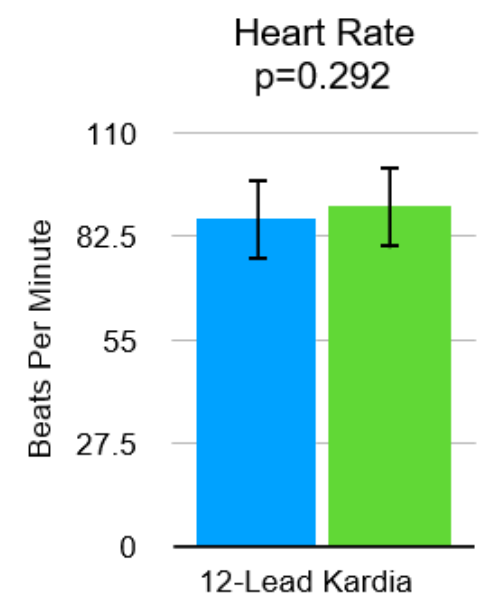

Figure 4: PR Interval and Heart Rate. 


\section{Limitations}

\section{Sample size and power}

As this is only a pilot study, the number was relatively low $(n=30)$. Generating a statistically significant difference in some of the metrics would have therefore been unlikely, unless drastically different. Therefore, this study may be subject to a type 2 error (the non- rejection of a false null hypothesis).

\section{Manual calculations}

Unlike the 12-lead ECG, Kardia does not automatically generate the QTc and PR intervals (it does automatically calculate heart rate). Clinicians, therefore, had to manually calculate QTc and PR intervals. This is simple to do, however it is somewhat time consuming and open to human error. The time to manually calculate these data points was not factored in.

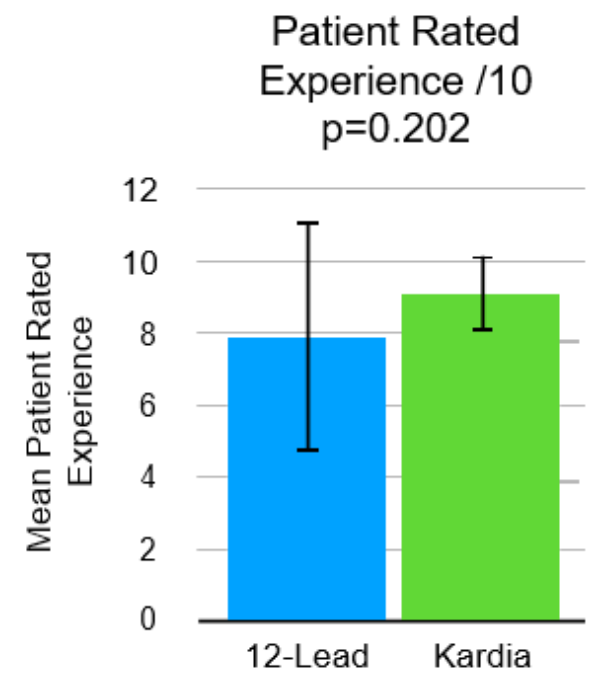

Figure 5: Subjective Patient Rated Experience.

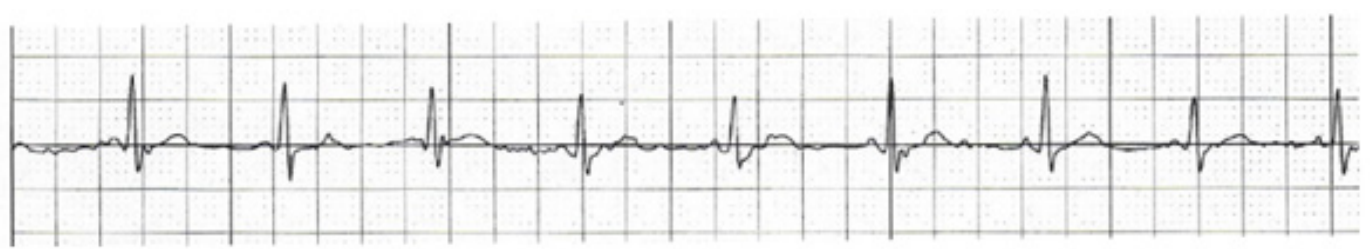

Figure 6: A small segment of a typical Kardia ECG.

\section{Ease of interpretation}

The Kardia ECG tracing was subject to more interference than a standard ECG, sometimes making interpretation more difficult. In one subject, we were unable to reliably interpret the Kardia ECG due to interference. This patient proceeded to have a 12-lead ECG.

\section{Further Development}

This pilot study shows very promising results. Kardia has been shown to be significantly quicker than the gold standard, while generating valid metrics. It would be useful to expand the study for a longer period of time or recruit further psychiatric patients. AliveCor have since released a new model which generates a 6 lead ECG. It would be interesting to see this in person and used in a similar trial.

\section{Acknowledgement}

None.

\section{Conflict of Interest}

No conflict of interest. 